\title{
diffusion-îundarrentals
}

The Open-Access Journal for the Basic Principles of Diffusion Theory, Experiment and Application

\section{Non-Fickian Interdiffusion of Dynamically Asymmetric Species: A Molecular Dynamics Study}

\author{
Jaklin Yaneva ${ }^{l}$, Burkhard Dünweg ${ }^{2}$, and Andrey Milchev ${ }^{I}$ \\ ${ }^{1}$ Bulgarian Academy of Sciences, Institute of Physical Chemistry, G. Bonchev Str. Block \\ 11, 1113 Sofia, Bulgaria, Email: milchev@ipc.bas.bg \\ ${ }^{2}$ Max-Planck Institut für Polymerforschung, Ackermannweg 10, D-55128, Mainz, \\ Germany
}

\section{Introduction}

One particular instance of non-Fickian transport is Case II diffusion[1], which is usually observed in glassy polymers subjected to penetration by a low-molecular weight solvent. The most characteristic feature of this phenomenon is the development of a sharp front in the concentration profile, which advances linearly in time, and ahead of which the penetrant concentration is very low. The development of the linearly advancing front is rather easy to understand. The two decisive preconditions are (i) the existence of a strong disparity in the mobilities of the two pure species (glassy matrix vs. penetrant), and (ii) the "plasticizing effect", i.e., a strong enhancement of the mobility of the slow species (constituent of the glassy matrix) if its molecules are surrounded by those of the fast species (penetrant). As soon as the slow molecules are plasticized at the front, they quickly make room for the penetrant, which in turn is rapidly transported into the thus opened free volume. This results in a spatially constant concentration profile behind the front. The rate of plasticization (matrix dissolution) is determined by the penetrant concentration at the front, and therefore remains constant. This gives rise to a constantvelocity front.

\section{Simulational model}

In our study we use Molecular Dynamics combined with Dissipative Particle Dynamics (DPD) to construct a model of a binary mixture where the two species differ only in their dynamic properties (friction coefficients). For an asymmetric mixture of slow and fast particles we study the interdiffusion process. The relaxation of the composition profile is investigated in terms of its Fourier coefficients. While for weak asymmetry we observe Fickian behavior, a strongly asymmetric system exhibits clear indications of anomalous diffusion, which occurs in a crossover region between the Cases I (Fickian) and II (sharp front moving with constant velocity), and is close to the Case II limit. As our results indicate, our model, though very simple, seems to grasp the main features of non-Fickian diffusion at least qualitatively. In contrast, recent attempts[2] to model computationally Case II diffusion did not observe any deviation from Fickian behavior, probably because the simulations failed to reach the necessary separation of time scales, even though the polymers were able to vitrify. We believe that a combination of our approach with a polymer model would prove promising in revealing the molecular aspects of anomalous diffusion in glassy polymers. The purpose of our study is hence twofold: On the one hand, we wish to further corroborate the view that dynamic disparity 
is indeed the decisive ingredient for Case II behavior. On the other hand, we propose a methodological advancement in the microscopic simulation of such phenomena.

\section{Conclusion}

Our computer simulations demonstrate that Case II behavior during the process of interdiffusion of two species can be observed in a model system where the two kinds of particles differ only in their dynamics. In contrast to previous simulation attempts, the approach to implement the dynamics contrast via vastly different DPD friction parameters seems to be particularly successful. Furthermore, the data analysis in terms of Fourier coefficients which take into account the finite system size and the boundary conditions has proven quite useful. In our model, the main feature of a system in which Case II diffusion can be observed is the difference in momentum relaxation of slow particles in the matrix and in the solvent, while the variation of the dynamics of the fast particles only slightly modifies the process of interdiffusion. It is clear, however, that more work is required before a clear-cut microscopic picture of anomalous diffusion emerges.

\section{References}

[1] J. Crank, The mathematics of diffusion, (Oxford University Press, Oxford, 1956). Ch. 11, p. 254

[2] M. Tsige and G. S. Grest, J. Chem. Phys. 120, 2989(2004); ibid. 121, 7513(2004).

[3] J. Yaneva, B. Dünweg, and A. Milchev, J. Chem. Phys. 122, May issue(2005), in press. 